\title{
Treatment of tobacco dependence in UK hospitals: an observational study
}

\author{
Authors: John Hutchinson, ${ }^{\mathrm{A}}$ Zaheer Mangera, ${ }^{\mathrm{B}}$ Laura Searle, ${ }^{\mathrm{C}}$ Anna Lewis ${ }^{\mathrm{D}}$ and Sanjay Agrawal ${ }^{\mathrm{E}}$ on behalf of the \\ British Thoracic Society
}

\begin{abstract}
Over a million smokers are admitted to hospitals in the UK each year. The extent to which tobacco dependence is identified and addressed in this population is unclear. Data on 14,750 patients from 146 hospitals collected for the British Thoracic Society smoking cessation audit were analysed to determine smoking prevalence, attempts to ask smokers about quitting, and referrals to smoking cessation services. Associations with hospital organisational factors were assessed by logistic regression. Overall hospital smoking prevalence was $25 \%$. Only $28 \%$ of smokers were asked whether they would like to quit, and only one in 13 smokers was referred for treatment of tobacco dependence. There was a higher chance of smokers being asked about quitting in organisations with smoke-free sites, dedicated smoking cessation practitioners, regular staff training, and availability of advanced pharmacotherapy. Treatment of tobacco dependence in smokers attending UK hospitals is poor and could be associated with organisational factors.
\end{abstract}

KEYWORDS: Smoking cessation, tobacco dependence, hospital, smoke-free

\section{Introduction}

Despite significant progress in reducing smoking prevalence over the past 30 years, almost $16 \%$ of the population of the UK still smoke, and around 100,000 deaths each year are attributable to smoking. ${ }^{1}$ The contribution of smoking to respiratory disease is not in doubt; ${ }^{2,3}$ however, non-respiratory conditions that are caused or exacerbated by tobacco use are also common, including ischaemic heart disease, cerebrovascular disease, cancers and infections, ${ }^{4}$ and many of these patients might be admitted to hospital for treatment.

Over a million smokers are treated in hospital each year in England alone, ${ }^{5}$ and there is evidence that hospital admission

Authors: ${ }^{A}$ consultant physician, Sherwood Forest Hospitals, Nottinghamshire, UK; ${ }^{\mathrm{B}}$ consultant physician, North Middlesex Hospital, London, UK; ' manager, Quality Improvement and Clinical

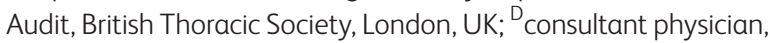
Cwm Taf University Health Board, Wales, Abercynon, UK; ${ }^{E}$ Consultant physician, Institute for Lung Health, Glenfield Hospital, Leicester, UK offers a prime opportunity to treat tobacco dependence. ${ }^{6}$ Increased contact with health professionals, perceived vulnerability because of illness or impending surgery, ${ }^{7,8}$ and time in a smoke-free environment away from usual triggers are among the reasons why smoking cessation might be more effective in hospitals. There is evidence from the UK that hospital-based smoking cessation interventions work. ${ }^{9}$ Furthermore, simply referring a smoker to community services might be less effective. ${ }^{10}$ Smoking cessation is important not only for the prevention of ill health, but also as a core treatment for many pre-existing conditions. Targeting cessation in hospital could reduce the length of stay, for example by avoiding poor wound healing after surgery. However, it is unclear whether clinicians are effectively targeting hospitalised smokers and actively seeking to address their tobacco dependence by using evidence-based interventions, including behavioural support and pharmacotherapy delivered by trained professionals. ${ }^{11}$ Therefore, in the current study, we sought to investigate the prevalence of smokers in UK hospitals, the extent to which these smokers were identified and offered treatment for their tobacco dependence, and the organisational determinants of treatment.

\section{Methods}

Clinicians from hospitals across the UK were invited to take part in the study through the 2016 British Thoracic Society (BTS) National Smoking Cessation Audit. Participating sites were instructed to review 100 randomly selected case notes of patients admitted in April and May 2016, to include at least 50 surgical and 50 medical admissions and covering at least two different specialties in each group. Patients from maternity, paediatric and mental health specialities were excluded. Participants were asked to screen each admission for smoking status, and to request additional records if fewer than 20 smokers were identified from the original selection.

For each record, information was collated on basic demographics (age and sex) as well as route of admission and hospital specialty. Documentation of smoking status, evidence that smokers were asked whether they would like to stop, and documentation as to onward referral and the use of licensed nicotine-containing products to help with abstinence were extracted from each record by local case-note reviewers. Further information on the organisation of local hospital-based stop-smoking services was also recorded; this included factors such as whether the hospital had a hospital smoking cessation practitioner, whether the 
service was supported by a dedicated senior member of staff and whether pharmacotherapy was available in the hospital. Additional questions covered areas such as whether designated smoking areas were provided and whether frontline staff (including junior doctors, nurses and pharmacists) were offered regular smoking cessation training.

In total, 146 hospitals contributed to the study, providing data on 14,750 patients. Data were collated locally and inputted into the national database via a web-based collection tool, with further analysis completed centrally. Guidance notes were provided to all organisations taking part, and the survey questions were piloted on a smaller cohort of hospitals before the main study. Data collection forms are available in the supplementary data file. The BTS Quality Improvement Committee determined that ethical approval was not required for the conduct of the audit.

This large data set was then used for the analysis. The main outcome measures were whether smoking was documented in the medical record, whether smokers were asked about quitting, and whether they were referred on to smoking cessation services. Hospital smoking prevalence figures were compared with national prevalence data for the UK obtained from the Office of National Statistics using the chi-squared test, ${ }^{1}$ which was also used to compare differences between demographic categories. Any patient classed as a current smoker without a valid record of smoking status from the current admission was not included in the analysis (smoking status likely obtained from elsewhere in the case notes, such as from preceding admissions). Logistic regression was used to assess the association between whether smokers were asked about quitting and both demographic and hospital organisational factors. Data analysis was performed using Stata, version 14.2 (StataCorp, TX, USA).

\section{Results}

Of the 14,750 patients screened, $73 \%$ had documentation of smoking status in the medical record. Of those with a valid record, $25 \%$ were current smokers. Demographics of the total population and the smoking population are shown in Table 1. Smoking prevalence was higher in males $(28 \%)$ than in females $(23 \%)$, with the highest prevalence in those less than 45 years of age (over $40 \%$ ). Smoking was most common in those admitted under respiratory medicine (30\%) and was more common in those admitted as emergencies (27\%) than as electives (19\%). The overall hospital smoking prevalence of $25 \%$ was significantly higher than the UK national average of 16\% ( $p<0.001)$.

Of 2,716 smokers, only 762 (28.1\%) had been asked whether they would like to quit, and only 153 (5.6\% of all smokers) had been referred to a hospital smoking cessation service. Overall, $57(2.1 \%)$ had been referred to community cessation services, $21(0.8 \%)$ had been referred back to their GP, and $15(0.6 \%)$ had been provided with self-referral information. However, 362 (13.3\%) reportedly 'did not want referral'. Of the smokers who had been asked whether they wanted to quit, 137 (18.0\%) had no further action documented.

Patients in the 46-55 and 56-65 age categories, and those admitted under respiratory medicine, were more likely to be asked whether they wanted to quit. Patients in Wales were less likely to be asked about quitting than were those in England, and emergency admissions were less likely to be asked than were elective admissions (Table 2).
In terms of organisation factors, there was a higher chance of being asked about quitting in institutions with access to hospitalbased smoking cessation services, those with a dedicated hospital smoking cessation practitioner, and those where services were supported by a dedicated senior member of staff (Table 3, Fig 1). Institutions providing regular training to frontline staff and those providing pharmacotherapy in addition to nicotine replacement therapy (varenicline and/or bupropion) also had a higher chance of being asked about quitting. Of the hospitals in the survey, $58 \%$ did not have varenicline on formulary. There was a negative association between being asked about quitting and whether an institution provided a designated smoking area, suggesting that staff in hospitals with smoking areas asked less about quitting. These associations persisted after adjusting for sex, age, country, route of admission and specialty.

\section{Discussion}

This is the largest study to date of the treatment of tobacco dependence in UK hospitals, reporting a smoking prevalence of $25 \%$ among those admitted to hospital, which is more than $50 \%$ higher than the overall UK prevalence of $15.8 \%$. Only $28 \%$ of smokers had been asked whether they wanted to quit, and only $28 \%$ of this number had been referred to hospital or community smoking cessation services, meaning that, overall, only one in 13 smokers had been referred for evidence-based treatment. There was an association between more-comprehensive hospital smoking cessation infrastructure (namely, smoke-free grounds, on-site smoking cessation practitioners, provision of regular staff training, and availability of comprehensive pharmacotherapy) and an increased chance of smokers being asked about quitting.

There is a gap in the literature on the prevalence of smoking in hospitals, but the results of this study suggest that a significantly higher proportion of people in hospital are smokers than among the general population. Smoking was particularly prevalent in younger patients, with prevalence rates remaining above $30 \%$ in those aged 65 or under, and among emergency admissions. Targeting smoking cessation treatment to hospitalised patients, a population enriched with patients who smoke, could have a significant impact as well as being an efficient use of resources. ${ }^{12}$

The high concentration of smokers in hospitals did not lead to high rates of treatment for tobacco dependence in our study, with only $28 \%$ of smokers being asked whether they would like to quit. This low rate of addressing tobacco smoking contrasts with evidence that hospital admission offers a prime opportunity to encourage quitting, ${ }^{6}$ thereby providing secondary prevention (to reduce disease progression and prevent exacerbations) and tertiary prevention (optimising function in the context of chronic illness). ${ }^{13-15}$ Evidence is also growing that smoking cessation in hospitalised patients is effective. ${ }^{9,12}$ The 'Ottawa Model' for smoking cessation, a systematic approach to tobacco dependence treatment used in Canadian hospitals, was recently shown to yield significantly lower rates of readmissions and emergency department attendances, as well as significant reduction in 1and 2-year mortality. ${ }^{12}$ The reasons for patients not being asked about quitting are likely multifactorial, but include beliefs that addressing smoking is too challenging or not a priority, and that patients might not want to quit, with smoking seen as a lifestyle choice rather than a medical problem that requires treatment. ${ }^{16-18}$ Despite this, most smokers do want to quit. ${ }^{19}$ People consume tobacco because of addiction to nicotine and the trigger of 
Table 1. Number of smokers by demographic status across UK hospitals

\begin{tabular}{|c|c|c|c|c|c|}
\hline Demographic status & Total sample (n [\%]) & $\begin{array}{l}\text { Proportion with valid } \\
\text { smoking status (\%) }\end{array}$ & Current smokers (n) & $\begin{array}{l}\text { Proportion of smokers } \\
\text { per group (\%) }\end{array}$ & $\mathrm{p}$-value \\
\hline \multicolumn{6}{|l|}{ Sex } \\
\hline Female & $7,476(50.7)$ & 71.4 & 1225 & 22.9 & \multirow[t]{2}{*}{$<0.001$} \\
\hline Male & $7,274(49.3)$ & 73.8 & 1491 & 27.8 & \\
\hline \multicolumn{6}{|l|}{ Age group (years) } \\
\hline $16-25$ & $842(5.7)$ & 72.0 & 251 & 41.4 & \multirow[t]{9}{*}{$<0.001$} \\
\hline $26-35$ & $1,047(7.1)$ & 71.0 & 308 & 41.5 & \\
\hline $36-45$ & $1,206(8.2)$ & 72.8 & 373 & 42.5 & \\
\hline $46-55$ & $1,754(11.9)$ & 74.3 & 487 & 37.4 & \\
\hline $56-65$ & $2,174(14.7)$ & 74.9 & 496 & 30.5 & \\
\hline $66-75$ & $3,047(20.7)$ & 76.3 & 494 & 21.3 & \\
\hline $76-85$ & $2,947(20.0)$ & 71.8 & 246 & 11.6 & \\
\hline $86-95$ & $1,618(11.0)$ & 64.6 & 59 & 5.7 & \\
\hline $96-105$ & $115(0.8)$ & 54.8 & 2 & 3.2 & \\
\hline \multicolumn{6}{|l|}{ Country } \\
\hline England & $13,532(91.7)$ & 72.4 & 2,482 & 25.3 & \multirow[t]{5}{*}{0.773} \\
\hline Scotland & $100(0.7)$ & 95.0 & 24 & 25.3 & \\
\hline Wales & $794(5.4)$ & 74.9 & 150 & 25.2 & \\
\hline Northern Ireland & $221(1.5)$ & 62.0 & 41 & 29.9 & \\
\hline Channel Islands & $103(0.7)$ & 80.6 & 19 & 22.9 & \\
\hline \multicolumn{6}{|l|}{ Route of admission } \\
\hline Elective & $3,419(23.2)$ & 68.7 & 446 & 19.0 & \multirow[t]{2}{*}{$<0.001$} \\
\hline Emergency & $11,331(76.8)$ & 73.8 & 2,270 & 27.2 & \\
\hline \multicolumn{6}{|l|}{ Specialty } \\
\hline Surgical specialty & $6,230(42.2)$ & 69.0 & 1,073 & 25.0 & \multirow[t]{5}{*}{$<0.001$} \\
\hline Other medical specialty & $6,063(41.1)$ & 74.7 & 1,097 & 24.2 & \\
\hline Respiratory medicine & $1,766(12.0)$ & 80.9 & 429 & 30.0 & \\
\hline Other/not known & $691(4.7)$ & 65.1 & 117 & 26.0 & \\
\hline Total - All patients & 14,750 & 72.6 & 2,716 & 25.4 & \\
\hline
\end{tabular}

dopaminergic pathways in the central nervous system. The rapid delivery of nicotine to the brain after inhalation of cigarette smoke contributes to its effectiveness, and this reinforces smoking behaviour as a means of satisfying nicotine cravings. ${ }^{20,21}$ This supports the concept of 'tobacco dependence' as a condition requiring treatment rather than simply a personal choice, and patients with high levels of nicotine addiction (eg those with mental health problems) benefit from treatment if it is offered. ${ }^{22}$

The associations we identified between organisational factors and asking about quitting are complex, but do suggest that institutions with more-proactive stop smoking services seize more opportunities to address cessation, and it is entirely plausible that a culture where stopping smoking is seen as a priority, with drivers including smoke-free hospital sites, will encourage staff to address this more readily. ${ }^{12}$ An example is the national smoke-free public places legislation in the UK, which came into force in 2007 and has contributed to a change in culture in several areas, including restaurants, transport and public buildings, with a significant decline in UK smoking prevalence and a reduction in passive smoke exposure in adults and children. ${ }^{23}$ Smoke-free hospital sites could encourage hospitals to treat tobacco dependence among inpatients more actively, reducing the impulse of patients to smoke inside hospital grounds. Although some institutions, such as mental health hospitals, face particular challenges with regards to smoke-free environments, there is evidence that policies can be implemented successfully, ${ }^{24-26}$ thereby reducing exposure of patients and staff to environmental tobacco smoke, ${ }^{27}$ with significant health benefits. ${ }^{28}$

Guidance from the UK National Institute for Health and Care Excellence (NICE) strongly supports the provision of hospitalbased stop-smoking services, with intensive behavioural support and early provision of pharmacotherapy to both inpatients and outpatients. ${ }^{11}$ There is evidence from both the UK and abroad that inpatient treatment of tobacco dependence increases quit rates and reduces readmissions. ${ }^{9,12}$ The National Institute for Health and Care Excellence recommends that all hospitals offer 
Table 2. Association between being asked about quitting and demographic factors

\section{Demographic factor} Current smokers

(n)

1,225

1,491

$07(27.3)$

Male

Age group (years)

$16-25$

26-35

$36-45$

46-55

56-65

66-75

$76-85$

$>85$

Country

England

Scotland

Wales

Northern Ireland

Channel Islands

Route of admission

Elective

Emergency

\section{Specialty}

Surgical specialty

Other medical specialty

Respiratory medicine

Other/not known

$\begin{array}{ll}251 & 53(21.1) \\ 308 & 69(22.4) \\ 373 & 83(22.3) \\ 487 & 144(29.6) \\ 496 & 178(35.9) \\ 494 & 158(32.0) \\ 246 & 69(28.1) \\ 61 & 8(13.1)\end{array}$

2,482

24

150

41

19

446

$146(32.7)$

2,270

1,073

1,097

429

117

$30(20.0)$

$7(36.8)$

$616(27.1)$

281 (25.6) (n (\%))
Asked about quitting? Unadjusted odds ratio (95\% CI)

1.00

$0.92(0.78-1.09)$

1.00

$0.92(0.77-1.09)$

0.314

Adjusted odds ratio

$p$ value $(95 \% \mathrm{CI})^{\mathrm{a}}$

1.00

1.00

$1.08(0.72-1.62)$

$1.01(0.67-1.53)$

0.952

$1.07(0.72-1.58)$

$1.01(0.68-1.49)$

0.973

1.57 (1.09-2.25)

$1.47(1.02-2.12)$

0.039

2.09 (1.47-2.98)

$1.80(1.25-2.59)$

0.001

$1.76(1.23-2.51)$

$1.43(0.99-2.06)$

0.059

$1.46(0.97-2.20)$

$1.17(0.77-1.79)$

0.460

$0.56(0.25-1.26)$

$0.44(0.20-1.01)$

0.051

1.00

1.00

$0.11(0.01-0.81)$

$0.11(0.01-0.82)$

0.031

$0.62(0.41-0.94)$

$0.60(0.39-0.91)$

0.016

$1.29(0.67-2.48)$

$1.07(0.55-2.10)$

0.842

$1.46(0.57-3.71)$

$1.34(0.51-3.50)$

0.548

1.00

1.00

$0.77(0.62-0.95)$

$0.68(0.53-0.87)$

0.002

1.00

1.00

$1.06(0.87-1.29)$

$1.17(0.95-1.46)$

0.140

$2.29(1.81-2.90)$

2.51 (1.93-3.27)

$<0.001$

$1.31(0.28-0.37)$

$1.26(0.82-1.94)$

0.284

Adjusted for sex, age, country, route of admission and admitting specialty. CI = confidence interval

a range of licensed nicotine replacement therapies, as well as varenicline (a partial nicotinic receptor agonist) and bupropion (an antidepressant), both of which have strong evidence of effectiveness above placebo in smoking cessation, with varenicline in particular among the most effective treatment options. ${ }^{29}$ The National Institute for Health and Care Excellence also specifically recommends provision of regular training to all frontline staff, as a means of ensuring all smokers are offered treatment for their tobacco dependence. ${ }^{11}$ The health economic benefits of treating tobacco dependence for individuals, hospitals and the wider society are well studied. In England, smoking is estimated to cost the NHS approximately $£ 2$ billion per year, ${ }^{4}$ and there is evidence that smoking cessation interventions in secondary care are costeffective. ${ }^{30}$ It is widely accepted that smoking cessation provides better value than many other interventions: as an example, the cost per quality-adjusted life year of stop-smoking support in chronic obstructive pulmonary disease is around a quarter of that of a single long-acting bronchodilator. ${ }^{31}$ Unfortunately, smoking cessation services in the UK are under threat because of cuts to local authority funding, and there have been calls for new approaches for funding and a re-emphasis that all opportunities should be taken to encourage smoking cessation in the $\mathrm{NHS}^{32}$

A key strength of the current study lies in the large sample size, covering a variety of hospitals from across the UK, including both medical and surgical specialties and both elective and emergency admissions. Therefore, the prevalence estimate is likely to be highly applicable to the UK, and an improvement on previous work based on large data sets without clinical validation. However, there are limitations to the observational study design, such as the possibility of response bias because of the variable enthusiasm of local specialists. Those who are frustrated with local services might downplay their provision, whereas others might overestimate services to deflect perceived criticism. The association between organisational factors and asking about quitting also needs interpreting with caution because of the possibility of confounding by additional factors, such as funding. Our assessment of case notes assumed that discussions about quitting smoking were adequately documented in medical records 
Table 3. Association between being asked about quitting and organisational factors

\begin{tabular}{|c|c|c|c|c|c|}
\hline \multicolumn{2}{|l|}{ Association } & $\begin{array}{l}\text { Asked about quitting? } \\
\text { (n [\%]) }\end{array}$ & $\begin{array}{l}\text { Unadjusted odds ratio } \\
(95 \% \mathrm{CI})\end{array}$ & $\begin{array}{l}\text { Adjusted odds ratio }{ }^{a} \\
(95 \% \mathrm{CI})\end{array}$ & p-value \\
\hline \multicolumn{6}{|c|}{ Does the trust have access to a hospital-based smoking cessation service? } \\
\hline \multicolumn{2}{|l|}{ No } & $184(18.1)$ & 1.00 & 1.00 & \\
\hline \multicolumn{2}{|l|}{ Yes } & $523(36.3)$ & $2.58(2.13-3.13)$ & $3.03(2.47-3.72)$ & $<0.001$ \\
\hline \multicolumn{6}{|c|}{ Does the trust have a dedicated hospital smoking cessation practitioner? } \\
\hline \multicolumn{2}{|l|}{ No } & $272(21.8)$ & 1.00 & 1.00 & \\
\hline \multicolumn{2}{|l|}{ Yes } & $438(35.3)$ & $1.96(1.64-2.35)$ & $2.30(1.91-2.78)$ & $<0.001$ \\
\hline \multicolumn{6}{|c|}{ Is the trust's smoking cessation service supported by a dedicated senior member of staff? } \\
\hline \multicolumn{2}{|l|}{ No } & $273(23.0)$ & 1.00 & 1.00 & \\
\hline \multicolumn{2}{|l|}{ Yes } & 437 (33.5) & $1.68(1.41-2.01)$ & $2.00(1.66-2.42)$ & $<0.001$ \\
\hline \multicolumn{6}{|c|}{ Does the trust offer additional pharmacotherapy to NRT on formulary? } \\
\hline \multirow[t]{2}{*}{ Bupropion (Zyban) } & No & $533(27.5)$ & 1.00 & 1.00 & \\
\hline & Yes & $177(32.3)$ & $1.26(1.03-1.55)$ & $1.25(1.01-1.54)$ & 0.041 \\
\hline \multirow[t]{2}{*}{ Varenicline (Champix) } & No & $346(24.2)$ & 1.00 & 1.00 & \\
\hline & Yes & $364(34.4)$ & $1.65(1.38-1.97)$ & $1.73(1.44-2.08)$ & $<0.001$ \\
\hline \multicolumn{6}{|c|}{ Does the trust have a dedicated smoking area? } \\
\hline \multicolumn{2}{|l|}{ No } & $473(30.8)$ & 1.00 & 1.00 & \\
\hline \multicolumn{2}{|l|}{ Yes } & $237(24.9)$ & $0.75(0.62-0.90)$ & $0.68(0.56-0.82)$ & $<0.001$ \\
\hline \multicolumn{6}{|c|}{ Does the trust offer frontline hospital staff regular smoking cessation training? } \\
\hline \multicolumn{2}{|l|}{ No (or not known) } & $341(24.2)$ & 1.00 & 1.00 & \\
\hline \multicolumn{2}{|l|}{ Yes } & $369(34.1)$ & $1.62(1.36-1.93)$ & $1.84(1.52-2.21)$ & $<0.001$ \\
\hline
\end{tabular}

and that these data were uniformly extracted, although a multisite pilot study completed in advance did attempt to anticipate potential problems. Furthermore, documentation of referral to stop-smoking services does not guarantee that patients actually attended these services and subsequently quit smoking, but it was necessary to use onward referral to specialists as a surrogate for being offered evidence-based treatment because this is the treatment that stop-smoking services provide.

\section{Conclusion}

Smoking is more common in secondary care than in the general population, but referral for treatment of tobacco dependence is rare. Smoke-free hospital grounds, on-site smoking cessation practitioners, regular staff training and the availability of advanced pharmacotherapy could improve treatment referral rates in hospitals.

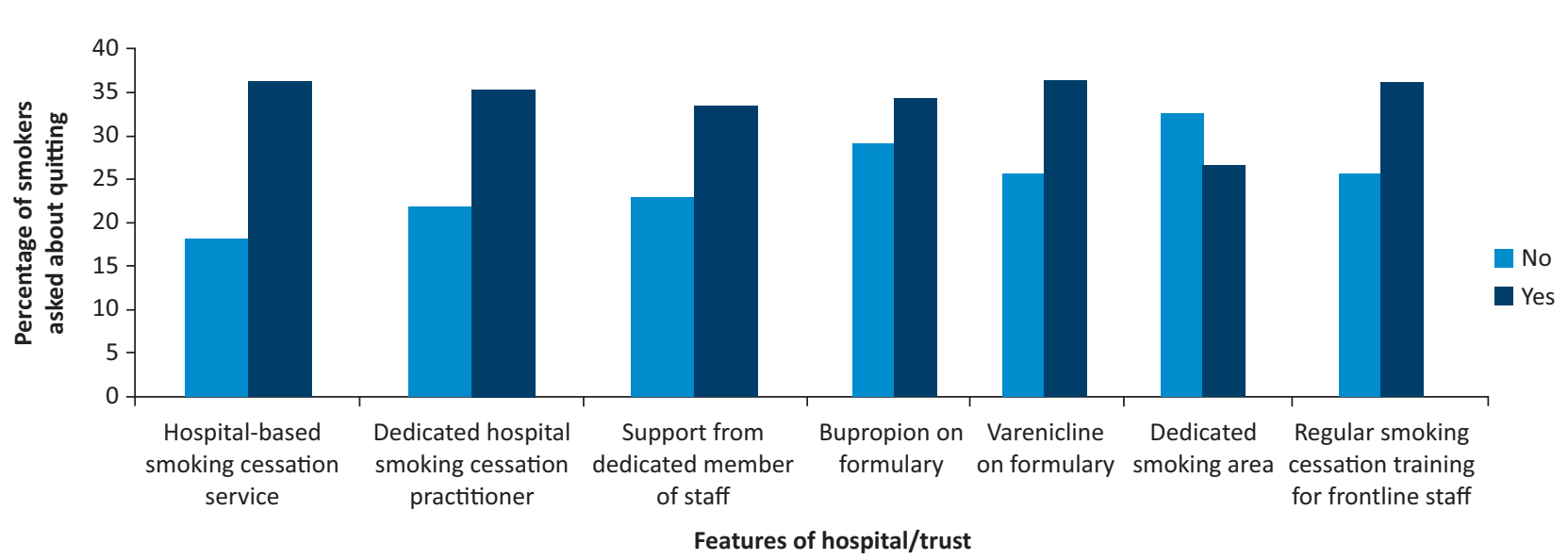

Fig 1. Association between trust organisational factors and likelihood of smokers being asked about quitting. 


\section{Conflicts of interest}

No conflicts of interest exist for this work.

\section{Author contributions}

The study was conceived by the authors and other members of the British Thoracic Society Tobacco Specialist Advisory Group. ZM, LS and SA designed the specific study questions, with input from others. All authors were involved in carrying out the study. JH wrote the first draft of the manuscript, with all authors involved in the revision and approval of the final draft. SA is the guarantor.

\section{Acknowledgements}

The authors acknowledge the support of the British Thoracic Society in collating the data for this study, as well as the clinicians and other staff across the country who participated in data collection.

\section{References}

1 Office of National Statistics. Adult smoking habits in the UK: 2016. London: Office of National Statistics, 2017.

2 Jayes L, Haslam PL, Gratziou CG et al. SmokeHaz: systematic reviews and meta-analyses of the effects of smoking on respiratory health. Chest 2016:150:164-79.

3 Jimenez-Ruiz CA, Andreas S, Lewis KE et al. Statement on smoking cessation in COPD and other pulmonary diseases and in smokers with comorbidities who find it difficult to quit. Eur Respir ] 2015;46:61-79.

4 Action on Smoking and Health. Smoking Still Kills. London; ASH, 2015.

5 Szatkowski L, Murray R, Hubbard R et al. Prevalence of smoking among patients treated in NHS hospitals in England in 2010/2011: a national audit. Thorax 2015;70:498-500.

6 Rigotti NA, Munafo MR, Stead LF. Smoking cessation interventions for hospitalized smokers: a systematic review. Arch Intern Med 2008;168:1950-60.

7 Twardella D, Loew M, Rothenbacher $\mathrm{D}$ et al. The diagnosis of a smoking-related disease is a prominent trigger for smoking cessation in a retrospective cohort study. J Clin Epidemiol 2006:59:82-9.

8 West R, Sohal T. "Catastrophic" pathways to smoking cessation: findings from national survey. BMJ 2006;332:458-60.

9 Murray RL, Leonardi-Bee J, Marsh ] et al. Systematic identification and treatment of smokers by hospital based cessation practitioners in a secondary care setting: cluster randomised controlled trial. BMJ 2013;347:f4004.

10 Lewis KE, Durgan L, Edwards VM et al. Can smokers switch from a hospital-based to a community-based stop smoking service? An open-label, randomized trial comparing three referral schemes. Nicotine Tob Res 2009;11:756-64.

11 NICE. Smoking cessation in secondary care: acute, maternity and mental health services (PH48). London: NICE, 2013.

12 Mullen KA, Manuel DG, Hawken S] et al. Effectiveness of a hospitalinitiated smoking cessation programme: 2-year health and healthcare outcomes. Tobacco Control 2017;26:293-9.

13 Public Health England and East Midlands Clinical Senate. Meeting the Prevention Challenge in the East Midlands - A Call to Action. London: NHS, 2015.
14 Thomson NC, Spears M. The influence of smoking on the treatment response in patients with asthma. Curr Opin Allergy Clin Immunol 2005;5:57-63.

15 Parsons A, Daley A, Begh R et al. Influence of smoking cessation after diagnosis of early stage lung cancer on prognosis: systematic review of observational studies with meta-analysis. BMJ 2010;340:b5569.

16 Caplan L, Stout C, Blumenthal DS. Training Physicians to Do Officebased Smoking Cessation Increases Adherence to PHS Guidelines. J Community Health 2011;36:238-43.

17 Tong EK, Strouse R, Hall J et al. National survey of U.S. health professionals' smoking prevalence, cessation practices, and beliefs. Nicotine Tob Res 2010;12:724-33.

18 Raupach T, Merker J, Hasenfuss $\mathrm{G}$ et al. Knowledge gaps about smoking cessation in hospitalized patients and their doctors. Eur ] Cardiovasc Prev Rehabil 2011:18:334-41.

19 Lader D. Opinions Survey Report No. 40 - Smoking related behaviour and attitudes, 2008/09. London: Office of National Statistics, 2009.

20 Royal College of Physicians. Nicotine without smoke - Tobacco harm reduction. London: RCP, 2016.

21 Benowitz NL. Nicotine addiction. N Engl J Med 2010;362:2295303.

22 Royal College of Physicians and Royal College of Psychiatrists. Smoking and mental health. London: RCP, 2013.

23 Royal College of Physicians. Passive smoking and children. London: RCP, 2010

24 Ratschen E, Britton J, McNeill A. Implementation of smoke-free policies in mental health in-patient settings in England. $\mathrm{Br}$ ] Psychiatry 2009;194:547-51.

25 Hashimoto K, Makinodan M, Matsuda Y et al. Smoking bans in mental health hospitals in Japan: barriers to implementation. Ann Gen Psychiatry 2015;14:35.

26 Arnott D, Wessely S, Fitzpatrick M. Should psychiatric hospitals completely ban smoking? BMJ 2015;351: h5654.

27 Etter M, Khan AN, Etter JF. Acceptability and impact of a partial smoking ban followed by a total smoking ban in a psychiatric hospital. Prev Med 2008:46:572-8.

28 Royal College of Physicians. Going smoke-free: the medical case for clean air in the home, at work and in public places. London: RCP 2005.

29 Hartmann-Boyce J, Aveyard P. Drugs for smoking cessation. BMJ 2016;352:i571.

30 Matrix NICE. Economic analysis of smoking cessation in secondary care. London: NICE, 2013.

31 British Thoracic Society. The case for change: why dedicated comprehensive and sustainable stop smoking services are necessary for hospitals. Br Thoracic Soc Rep 2013;5:1-18.

32 Cancer Research UK, Action on Smoking and Health. Cutting down: The reality of budget cuts to local tobacco control. London: CRUK/ASH, 2016.

Address for correspondence: Dr Sanjay Agrawal, Institute for Lung Health, Respiratory Biomedical Research Unit, Glenfield Hospital, Groby Road, Leicester LE3 9QP, UK.

Email: sanjay.agrawal@uhl-tr.nhs.uk 